\title{
O transplante de células-tronco hematopoéticas na leucemia linfoide crônica, uma proposta do I Encontro de Diretrizes do Transplante de Medula Óssea da Sociedade Brasileira de Transplante de Medula Óssea, Rio de Janeiro 2009
}

Hematopoietic stem cell transplantation in chronic lymphoid leukemia: a proposal by the Brazilian Consensus on Bone Marrow Transplantation of the Brazilian Society of Bone Marrow Transplantation, Rio de Janeiro 2009

Milton A. Ruiz ${ }^{1}$

José Carlos A. Barros ${ }^{2}$

Rodrigo Santucci ${ }^{3}$

Lilian P. Ruiz ${ }^{4}$

Denis Taveira

\begin{abstract}
Portadores de leucemia linfoide crônica (LLC) apresentam curso clínico indolente e prolongado que devem ser diferenciados daqueles que têm doença de evolução agressiva e fatal. Pacientes mais jovens e com critérios de alto risco podem se beneficiar com tratamento mais agressivo como o transplante de células-tronco hemopoéticas (TCTH). O transplante autólogo apresenta casos com remissão citogenética e molecular, baixa taxa de mortalidade, mas não demonstram platô nas curvas de sobrevivência e alta taxa de recaídas. Os transplantes alogênicos com regime mieloablativos têm altos índices de toxicidade e mortalidade, mas evidenciam o efeito enxerto versus leucemia, que aumenta a possibilidade de cura destes indivíduos. Assim, a opção dos transplantes alogênicos está dirigida para os transplantes com regime de condicionamento não mieloablativo, que pode ser aplicado inclusive a pacientes mais idosos ou portadores de comorbidades, e manter o potencial efeito GVL. A identificação dos pacientes que podem ser beneficiados por esses procedimentos, caracterizar e apontar os novos marcadores prognósticos permanece objeto de muitos estudos clínicos e foi o objetivo do grupo responsável em discutir as diretrizes do TCTH no consenso da Sociedade Brasileira de Transplante de Medula Óssea-SBTMO. Assim, consideramos que o TCTH para a leucemia linfoide crônica (LLC) deve seguir, para sua indicação, os critérios do European Group for Blood and Marrow Transplantation (EBMT) e, quando houver disponibilidade de um doador aparentado, a opção deve ser do TCTH alogênico com regime não mieloablativo. $O$ TCTH alogênico não aparentado e o autólogo devem ser considerados como opção secundária de indisponibilidade de doador, situações especiais e ensaios clínicos. Rev. Bras. Hematol. Hemoter. 2010;32(Supl. 1):91-96.
\end{abstract}

Palavras-chave: Leucemia linfoide crônica; transplante de células-tronco hematopoéticas; alogênico; autólogo; regimes de condicionamento; tratamento.

\footnotetext{
${ }^{I}$ Hematologista. Médico colaborador da disciplina de Hematologia da Faculdade de Medicina da Universidade de São Paulo (USP)-SP. ${ }^{2}$ Hematologista/Transplante de Medula Óssea. Coordenador do Serviço de TMO da Santa Casa de Misericórdia de São Paulo-SP. ${ }^{3}$ Hematologista. Médico Assistente do Serviço de TMO do Hospital Santa Paula - São Paulo-SP.

${ }_{5}^{4}$ Hematologista. Médica Assistente do Serviço de TMO do Hospital Infante D. Henrique da APB - São José do Rio Preto-SP.

${ }^{5}$ Hematologista. Médico colaborador do Serviço de TMO do Hospital Universitário Pedro Ernesto da Universidade do Estado do Rio de Janeiro (UERJ) - Rio de Janeiro-RJ.
}

Faculdade de Medicina da Universidade de São Paulo, FM USP - São Paulo-SP

Correspondência: Milton Artur Ruiz

Rua Catarina Nucci Parise, 760 - Jardim Vivendas

15090-470 - São José do Rio Preto-SP - Brasil

E-mail: milruiz@yahoo.com.br

Doi: 10.1590/S1516-84842010005000018 


\section{Introdução}

A leucemia linfoide crônica do tipo B (LLC) é uma doença heterogênea de curso clínico comumente indolente, caracterizada por um progressivo acúmulo de linfócitos, habitualmente $\mathrm{CD} 5+$ nos tecidos linfoides, medula óssea e sangue periférico. A identificação da moléstia remonta a 1903, com Turk, e a descrição detalhada da LLC em oitenta casos foi realizada por Minot e Isaacs, em 1924. ${ }^{1}$ A LLC acomete os indivíduos idosos, sendo a mediana de idade do aparecimento da doença ao redor dos 65 anos. A maioria dos pacientes tem idade acima dos 60 anos, mas 1/3 destes está abaixo desta idade. A ocorrência de casos abaixo dos 50 anos é considerada rara, sendo extremamente inusitado o seu aparecimento abaixo dos 40 anos de idade. ${ }^{1}$ É o tipo de leucemia mais frequente nos adultos ocidentais, rara nos orientais e duas vezes mais frequente entre os homens..$^{2-4}$ Ocorre aproximadamente de três a cinco casos de LLC anuais para cada 100 mil habitantes. ${ }^{1} \mathrm{~A}$ incidência anual para pacientes acima de 70 anos é de vinte casos por 100 mil habitantes. ${ }^{3}$ Existem descrições de aumento do número de casos da moléstia. Este dado pode ser creditado a vários fatores, tais como o hábito recente da população de realizar exames periódicos, aprimoramento dos recursos de diagnóstico e a mudança nos critérios de diagnóstico. ${ }^{5}$ A etiologia da LLC é desconhecida, existindo, porém, casos entre irmãos, parentes e até mesmo em diversas gerações de uma mesma família. ${ }^{6-8} \mathrm{Não}$ existem evidências de responsabilidade de agentes ambientais na gênese da moléstia ${ }^{8,9}$ ou de relato de casos em expostos a radiações ionizantes,${ }^{10}$ agentes químicos, derivados de petróleo ou a carcinogênicos clássicos como o benzeno. ${ }^{9-11}$ Foi demonstrada uma associação entre LLC e o "agente laranja," herbicida desfolhante utilizado durante a guerra do Vietnam entre 1962 a $1971 .{ }^{12}$ A LLC expressa altos níveis de proteínas antiapoptóticas, como as da família BCL, e baixos níveis de proteínas pró-apoptóticas, como a BAX. ${ }^{13}$ Nenhum dos proto-oncogenes habitualmente envolvidos nas neoplasias malignas de células B, como o BCL 2, BCL 6, PAX- 5 ou C- MYC encontram-se alterados na LLC. ${ }^{14}$

As alterações citogenéticas estão presentes em mais de $80 \%$ dos casos, e associadas a alterações moleculares conferem fatores prognósticos que podem ser utilizados na prática clínica. ${ }^{15}$

\section{Fatores de prognóstico}

São considerados fatores de prognóstico os sistemas de classificação de Rai e Binet, que utilizam dados clínicos e hematológicos, apresentam grande importância e têm o condão de predizer e nortear a abordagem terapêutica (Tabela 1). São fatores de prognóstico, além dos sistemas de classificação, a duplicação linfocitária no período de 12 meses, ${ }^{16} \mathrm{o}$ padrão histopatológico de comprometimento da medula óssea, ${ }^{17}$ os níveis séricos elevados da $\beta 2$-microglobulina ${ }^{18}$ do
Tabela 1. Estadiamentos clínicos da leucemia linfoide crônica

\begin{tabular}{|c|c|c|c|}
\hline Estádio & $\begin{array}{l}\text { Dados clínicos e } \\
\text { laboratoriais }\end{array}$ & Risco & $\begin{array}{r}\text { Sobrevida } \\
\text { média/anos }\end{array}$ \\
\hline \multicolumn{4}{|l|}{ Rai } \\
\hline 0 & Linfocitose isolada & Baixo & 14,5 \\
\hline I & Linfocitose, adenopatias & intermediário & 7,5 \\
\hline II & $\begin{array}{l}\text { Linfocitose, } \\
\text { esplenomegalia e/ou } \\
\text { hepatomegalia }\end{array}$ & intermediário & 7,5 \\
\hline III & $\begin{array}{l}\text { Linfocitose, anemia } \\
\qquad(\mathrm{Hb}<11,0 \mathrm{~g} / \mathrm{dl})\end{array}$ & Alto & 2,5 \\
\hline IV & $\begin{array}{c}\text { Linfocitose, } \\
\text { trombocitopenia } \\
\text { (Plaquetas }<100 \times 10\end{array}$ & alto & 2,5 \\
\hline \multicolumn{4}{|l|}{ Binet } \\
\hline A & $\begin{array}{l}\text { Linfocitose, menos de } \\
3 \text { áreas de } \\
\text { envolvimento linfoide }\end{array}$ & & 14 \\
\hline$B$ & $\begin{array}{l}\text { Linfocitose, } 3 \text { ou mais } \\
\text { áreas de envolvimento } \\
\text { linfoide }\end{array}$ & & 5 \\
\hline $\mathrm{C}$ & $\begin{array}{l}\text { Comprometimento da } \\
\text { função medular - } \\
\text { anemia, trombocitopenia, } \\
\text { +A ou B ou ambos }\end{array}$ & & 2,5 \\
\hline
\end{tabular}

antígeno $\mathrm{C} 23$ solúvel ${ }^{19}$ e da timidina quinase. ${ }^{20,21}$ As alterações citogenéticas observadas nos pacientes com LLC podem subdividir os pacientes em grupos de risco, e determinadas alterações podem predizer os pacientes com mais chance de uma má evolução. ${ }^{22-25}$ As alterações em $17 \mathrm{p}$ e 11q têm prognóstico desfavorável, assim como a do gene de supressão tumoral $p 53 .{ }^{26}$ Alteração do $p 53$ é considerada um dos fatores de prognósticos adversos dos mais importantes segundo análise multivariada em estudos prospectivos e retrospectivos com pacientes nas fases iniciais ou avançadas da LLC. Foi observada que a incidência de anormalidades é baixa em pacientes assintomáticos nas fases iniciais da doença, existindo sugestões de terapia precoce em pacientes que sejam portadores desta anormalidade. ${ }^{27}$

O gene da imunoglobulina (Ig) tem um papel importante na patogenia da moléstia e os rearranjos da região variável humana (VH) são considerados preditores. ${ }^{28}$ Os pacientes com ausência de mutação no $I g V H$ têm mal prognóstico em relação aos pacientes que apresentam a mutação. Aparentemente existe uma correlação entre alteração do status mutacional do $I g V H$ e aberração genômica. ${ }^{29}$ A expressão do CD38 está presente em percentual ao redor de $20 \%$ dos pacientes e os que a expressam apresentam redução na sobrevida global e uma aparente correlação com o status mutacional do gene $I g V H .{ }^{28-33} \mathrm{O}$ ZAP-70 (Proteína Zeta Associada de $70 \mathrm{kDa}$ ) é uma proteinocinase que normalmente está expressa nos linfócitos T e NK e é detectada com a citometria de fluxo. ${ }^{34}$ Nos pacientes portadores de LLC, o 
ZAP-70 pode ou não estar aumentado e tem correlação com o status mutacional do $\operatorname{IgVH}$, progressão de moléstia e a sobrevida. ${ }^{34-36}$

São considerados de alto risco, segundo o EBMT, ${ }^{37} \mathrm{e}$ passíveis de serem utilizados para indicação do TCTH ou de terapias de segunda linha os critérios abaixo:

- Ausência de resposta ou recaída precoce, no prazo de 12 meses, após o tratamento com análogos da purina,

- Recaída, no prazo de 24 meses, após terapia de análogo da purina combinada com outro medicamento, ou tratamento de similar eficácia como é considerado o transplante de células-tronco hematopoéticas autólogo.

- Deleção do $p 53$ / mutação del 17p13 em paciente que necessite tratamento

- A indicação do TCTH autólogo deve estar indicada em conjunto a protocolos clínicos.

\section{Método}

Para obtenção destes dados foi realizada uma pesquisa bibliográfica no Medline e no Clinical Trials do National Institute of Health, USA, seguindo as orientações da organização do consenso de TMO da SBTMO.

Todos os participantes tiveram igual participação na elaboração deste documento, e esta é a versão final adaptada após o primeiro encontro de diretrizes sobre o transplante de medula óssea realizada no Rio de Janeiro em junho de 2009.

Na sequência discutiremos as várias modalidades de transplante, regimes de condicionamento, os resultados e status atual do TCTH.

\section{Transplante de células-tronco hematopoéticas autólogo}

Estudos têm demonstrado obtenção de remissão clínica e molecular com o TCTH autólogo em pacientes com LLC de alto risco ou refratários aos tratamentos convencionais. ${ }^{38,39}$ Os resultados observados são em estudos de braço único ou comparativo, não randomizados, e, portanto, limitados. Não existem publicações de estudos prospectivos controlados comparando TCTH autólogo versus quimioterapia. Nenhum desses trabalhos também conseguiu demonstrar um platô nas curvas de sobrevida global. A viabilidade do TCTH foi demonstrada desde 1993 quando foram avaliados vinte pacientes portadores de LLC de alto risco, condicionados com radioterapia corporal total (TBI) e ciclofosfamida, que, na sequência, receberam resgate autólogo, com células purgadas com anticorpos monoclonais, ou receberam resgate alogênico depletados de linfócitos T. Os resultados observados foram: remissão completa (RC) em $89 \%$ e baixa mortalidade relacionada ao transplante (MRT) evidenciando que o procedimento era seguro e, a partir de então, devia ser considerado como mais uma opção terapêutica para a LLC..$^{40}$
Estudo de "coorte" europeu ${ }^{38}$ demonstrou melhora da sobrevida em pacientes de alto risco submetidos ao TCTH autólogo quando comparado a um grupo tratado somente com quimioterapia convencional, o que possibilitava a conclusão de que essa opção de tratamento poderia oferecer melhores perspectivas a esses indivíduos. Muitas dúvidas surgiram, tais como a de definir o melhor momento de se indicar o TCTH, valorização dos fatores prognósticos que realmente teriam significado para essas indicações, e quais seriam os melhores regimes de condicionamentos. Outra dúvida seria o de determinar o papel dos purging tanto in vivo quanto in vitro, e como esses pacientes deveriam ser seguidos e monitorizados após o procedimento. Análises europeias enfatizam a indicação do TCTH autólogo mais precocemente, evitando-se assim o efeito adverso cumulativo de quimioterapias no tratamento de recaídas. ${ }^{41,42}$ A maioria destas publicações tem seguimento relativamente curto e estão mais direcionadas para a avaliação da MRT do TCTH autólogo. O desenvolvimento secundário de mielodisplasias, leucemia mieloide aguda, ou mesmo de tumores sólidos em curto período de seguimento, são fatores de preocupação principalmente nos protocolos que envolvem TBI e considerando que a LLC, de forma geral, tem curso natural longo. ${ }^{43-46}$

\section{Transplante de células-tronco hematopoéticas alogênico mieloablativo}

Os objetivos principais dos TCTH alogênicos em pacientes portadores de LLC são:

- Infusão de células sadias (sem neoplasia) proveniente de doador igualmente sadio.

- Obtenção do efeito imunológico do enxerto versus leucemia $(\mathrm{GVL})$, e assim diminuir o número de recaídas da doença.

Entretanto, este procedimento tem alta taxa de morbidade, e a MRT é elevada, principalmente devido à idade avançada observada na maioria dos pacientes com LLC, ${ }^{47}$ infecções secundárias e pela doença do enxerto contra hospedeiro $(\mathrm{DECH})$. Dados do Registro Europeu indicam uma MRT de $46 \%$, sendo a mortalidade secundária à DECH de $20 \%{ }^{48}$ Estes números se assemelham ao observado no Fred Hutchinson Cancer Center, onde a mortalidade não associada a recidivas foi de $57 \%$ (primeiros cem dias), principalmente nos pacientes que receberam condicionamento com bussulfano.$^{49}$ Dados de uma publicação canadense também ressaltam um potente efeito GVL em pacientes que apresentam DECH (aguda e crônica).

Não existem também estudos prospectivos randomizados controlados comparando o TCTH autólogo versus o TCTH alogênico. Estudo do M.D. Anderson Cancer Center demonstra melhores resultados do TCTH alogênico, com a indução de remissões mais prolongadas, mesmo em pacientes com doença refratária. ${ }^{50}$ Embora as recaídas sejam menores, a sobrevida de pacientes submetidos ao TCTH alogênico 
(principalmente nos que tiveram transplantes não aparentados) é reduzida basicamente pela toxicidade do procedimento em pacientes com idade avançada, em doença de alto risco ou refratárias. Estes dados suportam a necessidade de serem utilizados regimes de condicionamentos com doses menores dos agentes quimioterápicos, mas mantendo o objetivo de preservação do efeito GVL. ${ }^{47}$

\section{Transplantes alogênicos não mieloablativos ou de intensidade reduzida}

A possibilidade de drástica redução da elevada MRT do TCTH alogênico, mantendo a enxertia das células do doador, pode ser alcançada através da diminuição das doses dos agentes que participam dos regimes de condicionamento, embora os dados sejam ainda provenientes de poucos ensaios clínicos. Os autores iniciais viram que os transplantes de células-tronco hematopoéticas alogênicos não mieloablativos de intensidade reduzida (RIC) podiam ser aplicados a pacientes com idades mais avançadas. Assim, foram observados dados mais completos em um maior número de pacientes portadores de LLC. Os estudos estão listados na Tabela 2. número elevado de quimioterapias prévias e citogenética adversa. ${ }^{56}$ Portanto, conclui-se que os RIC têm potencial efetivo de induzir remissões em pacientes portadores de LLC de alto risco. ${ }^{58,59}$

\section{Uso de anticorpos monoclonais nos RIC}

O uso de anticorpos monoclonais nestes procedimentos visa basicamente à redução da $\mathrm{DECH}$ sem comprometimento do efeito GVL. Resultados promissores foram obtidos no M.D. Anderson Cancer Center com a adição do rituximab ao condicionamento (fludarabina e ciclofosfamida). ${ }^{53}$

Já o uso de alemtuzumab nos condicionamentos, embora com maior atividade antitumoral, demonstrou um retardo na enxertia e aumento de complicações infecciosas com altas taxas de mortalidade sem que houvesse uma potencialização do efeito GVL.55

\section{Recomendações}

A obtenção da remissão clínica hematológica citogenética e molecular é objetivo do tratamento da LCC. O TCTH alogênico é considerado, mesmo com o aparecimento de novos medicamentos efetivos, como a única opção de cura para os portadores de LLC. O RIC é uma opção para um grupo maior de pacientes mais jovens, refratários ou com critérios de risco pelos marcadores (de risco) bioquímicos, citogenéticos ou moleculares. O TCTH autólogo deve constar do arsenal terapêutico para pacientes que não possuam doador, mas que apresentam fatores de prognóstico de risco como tratamento de consolidação em protocolos de estudos clínicos. A presença de doença residual mínima pode ser comprovada com a detecção do rearranjo do gene do IGVH e do ZAP70, sendo os mesmos considerados critérios de eleição para se determinar a evolução dos pacientes. A monitorização destes pacientes deve ser periódica e abranger, além dos dados clínicos e hematológicos, estudos citogenéticos e de imunofenotipagem medular.

\footnotetext{
Abstract

Patients with chronic lymphocytic leukemia usually have an indolent and prolonged clinical course and need to be differentiated from those who have an aggressive and fatal disease. Younger patients with high-risk criteria may benefit with a more aggressive treatment that includes hematopoietic stem cell transplantation (HSCT). Autologous transplantation, despite of the encouraging results with
} 
cases of molecular and/or cytogenetic remission and low mortality rates, does not present a plateau in survival curves and has a high relapse rate. Allogeneic transplantations using myeloablative regimens, have high toxicity and mortality rates, but also demonstrate the graft-versus-leukemia effect that increases the possibility of cure of these individuals. So the option of allogeneic transplants for patients with CLL is directed to conditioning using non-myeloablative regimens, which can also be applied to older patients or those with comorbidities, and maintain a potential graftversus-leukemia effect. The identification of patients who may benefit from these procedures and the characterization of new prognostic markers remain the subjects of many clinical studies and were the objective of the group responsible for discussing guidelines for CLL of the consensus on HSCT SBTMO. Thus we believe that HSCT for CLL should follow the criteria of the EBMT. When a sibling donor is available the best option is allogeneic HSCT with a myeloablative regimen. The strategy of unrelated allogeneic or autologous HSCT must be considered as a second option when no donor is available, for special situations and clinical trials. Rev. Bras. Hematol. Hemoter. 2010;32(Supl. 1):91-96.

Key words: Chronic lymphocytic leukemia; hematopoietic stem cells transplantation; allogeneic; autologous; conditioning regimen; treatment.

\section{Referências Bibliográficas}

1. Rai KR, Wasil T. History of chronic lymphocytic leukemia 1-7 in Cheson BD Chronic lymphoid leukemias. 2001, Marcel Dekker Inc. USA, 625p.

2. Dighiero G, Travade P, Chevret S, et al. B-cell chronic lymphocytic leukemia: present status and future directions. French Cooperative Group CLL. Blood. 1991;78(8):1901-14.

3. Caligaris-Cappio F, Hamblin TJ. B-cell chronic lymphocyte leukemia: a bird of different feather. J Clin Oncol. 1999;17(1): 399-408.

4. Ries LAG, Kosary CL, Hankey BF, et al. SEER Cancer statistics review, 1973-1999 NIH Pub No 99-2789 Bethesda, MD: National Cancer Institute, 1999:262-83

5. Diehl LF, Karnell LH, Menck HR. The American College of Surgeons Commission on Cancer and the American Cancer Society. The National Cancer Data Base report on age, gender, treatment, and outcomes of patients with chronic lymphocytic leukemia. Cancer. 1999;86(12):2684-92

6. Linet MS. Van Natta ML, Brookmeir R, et al. Familial cancer history and chronic lymphocytic leukemia. A case control study. Am J Epidemiol. 1989;130(4):655-64.

7. Fernhout F Dinkelaar RB, Hagemeijer A, et al. Four aged siblings with B cell chronic lymphocytic leukemia. Leukemia. 1997;11(12): 2060-5.

8. Crowther-Swanepoel D, Houlston R. The molecular basis of familial chronic lymphocytic leukemia. Haematologica 2009; 49(5):606-9.

9. Sgambati MT, Linet MS, Devesa SS. Chronic lymphocytic leukemia, epidemiological, familial, and genetic aspects 33-62 in Cheson BD "Chronic Lymphoid Leukemias. 2001. Marcel Dekker Inc. USA 625p.

10. Rinsky RA, Smith AB, Hornung R, et al. Benzene and leukemia. An epidemiologic risk. N Eng J Med. 1987;316(17):1044-50.

11. Utterback DF, Rinsky RA. Benzene exposure assessment in rubber hydrochloride workers: a critical evaluation of previous estimates. Am J Ind Med. 1995;27(5):661-76.
12. Marwick C. Link between Agent Orange and chronic lymphocytic leukaemia. BMJ. 2003;326(7383):242.

13. Gaidano G, Newcomb BEW, Gong JZ, et al. Analysis of alterations of oncogenes and tumor suppressor genes in chronic lymphocytic leukemia. Am J Path. 1994:144(6):1312-19.

14. Bullrich F, Croce CM. Molecular biology on chronic lymphocytic leukemia 9-25 in Cheson BD "Chronic lymphoid leukemias. 2001 Marcel Dekker Inc. USA 625p.

15. Gribben JG. Molecular profiling in CLL. Hematology Am Soc Hematol Educ Program. 2008:444-9.

16. Montserrat E, Sanchez-Bisono J, Vinolas N, Rozman C. Lymphocyte doubling time chronic lymphocyte: analysis of its prognostic significance. Br J Haematol. 1986;62(3):567-75.

17. Rozman C, Montserrat E, Rodrigues-Fernandez JM. Bone marrow histologic pattern - the best single prognostic parameter in chronic lymphocytic leukemia: a multivariate survival analysis of 329 cases. Blood. 1984;64(3):642-8.

18. Keating MJ, Lerner S, Kantarjian $\mathrm{H}$, et al. The serum beta 2 microglobulin level is more powerful than stage in predicting response and survival in chronic lymphocytic leukemia. Blood. 1996;88:4259-64.

19. Molica S, Levato D, Dell'Olio M, et al. Cellular expression and serum circulating levels of CD23 in B-cell chronic lymphocytic leukemia. Implications for prognosis. Haematologica. 1996;81 (15):428-33

20. Hallek M, Langenmayer I, Nerl C, et al. Elevated serum thymidine kinase levels identify a subgroup at high risk of disease progression in early, nonsmoldering chronic lymphocytic leukemia. Blood 1999;93(5):1732-37.

21. Zwiebel JA, Cheson BD. Chronic Lymphocytic Leukemia: staging and prognostic factors. Semin Oncol. 1998;25(1):42-59.

22. Juliusson G, Oscier DG, Fitchett M, et al. Prognostic subgroups in B-cell chronic lymphocytic leukemia defined by specific chromosomal abnormalities. N Engl J Med. 1990;323 (11): $320-4$.

23. Fegan C, Robinson H, Thompson P, et al. Karyotypic evolution in CLL: identification of a new sub-group of patients with deletions of $11 \mathrm{q}$ and advanced or progressive disease. Leukemia. 1995; 9(12):2003-8

24. Dönner H, Stilgenbauer S, James MR, et al. 11q deletions identify a new subset of B-cell chronic lymphocytic leukemia characterized by extensive nodal involvement and inferior prognosis. Blood. 1997;89(7):2516-22

25. Neilsen JR, Auer R, White D, et al. Deletions at 11q identify a subset of patients with typical CLL who show consistent disease progression and reduced survival. Leukemia. 1997;11(11):1929-32.

26. el Rouby S, Thomas A, Costin D, et al. p53 gene mutation in B cell chronic lymphocytic leukemia is associated with drug resistance and is independent of MDR1/MDR3 gene expression. Blood. 1993;82(11):3452-59.

27. Best OG, Gardiner AC, Mould SJ, et al. A subset of Binet stage A CLL patients with TP53 abnormalities and mutated IGHV genes have stable disease. Leukemia. 2009;23(1):212-4.

28. Damle RN, Wasil T, Fais F, et al. Ig V gene mutation status and CD38 expression as novel prognostic indicators in chronic lymphocytic leukemia. Blood. 1999;94(6):1840-47.

29. Hernández JA, Rodriguez AE, González M, et al. A high number of losses in $13 \mathrm{q} 14$ chromosome is associated with a worse outcome and biological differences in patients with B-cell chronic lymphocytic leukemia. Haematologica. 2009;94(3):364-71.

30. Ottagio L, Viaggi S, Zunino A, et al. Chromosome aberrations evaluated by comparative genomic hybridization in B-cell chronic 
lymphocytic leukemia: correlation with CD38 expression. Haematologica. 2003;88(7):769-77.

31. Ibrahim S, Keating M, Do KA, et al. CD38 expression as an important prognostic factor in B-cell chronic lymphocytic leukemia. Blood. 2001;98(1):181-6.

32. Thunberg U, Johnson A, Roos G, et al. CD38 expression is a poor predictor for $\mathrm{VH}$ gene mutational status and prognosis in chronic lymphocytic leukemia. Blood. 2001;97(6):1892-93.

33. Damle RN. Immunoglobulin V genes and CD38 expression analysis in B-cell chronic lymphocytic leukemia. Blood. 2001;97:1893-95.

34. Crespo M, Bosch F, Villamor N, et al. ZAP-70 expression as a surrogate for immunoglobulin-variable-region mutations in chronic lymphocytic leukemia. N Engl J Med. 2003;348(18): 1764-75.

35. Bosch F, Villamor N. ZAP-70 expression in CLL: a new parameter for an old disease. Haematologica 2003;88(7):724-5.

36. Reinoso-Martin C, Jantus-Lewintre E, Ballesteros E, et al. ZAP70 mRNA expression provides clinically valuable information in early-stage chronic lymphocytic leukemia. Haematologica 2008; 93(9)1422-24.

37. Dreger P. HSCT for Chronic lymphocytic leukemia in adults, 406413 in Haematopoietic Stem cell Transplatiox EBMT hand book 5 th ed. 2008, $591 \mathrm{p}$

38. Dreger P, Stilgenbauer S, Benner A, et al. The prognostic impact of autologous stem cell transplantation in patients with chronic lymphocytic leukemia: a risk-matched analysis based on the $\mathrm{VH}$ gene mutational status. Blood. 2004;103(7):2850-58.

39. Gribben JG, Zahrieh D, Stephans K, et al. Autologous and allogeneic stem cell transplantations for poor-risk chronic lymphocytic leukemia. Blood. 2005;106(13):4389-96.

40. Rabinowe SN, Soiffer RJ, Gribben JG, et al. Autologous and allogeneic bone marrow transplantation for poor prognosis patients with B-cell chronic lymphocytic leukemia. Blood. 1993; 82(4):1366-76.

41. Sutton L, Maloum K, Gonzalez H, et al. Autologous hematopoietic stem cell transplantation as salvage treatment for advanced B cell chronic lymphocytic leukemia. Leukemia. 1998;12(11):16991707.

42. Dreger P, Busch R, Stilgenbauer S, et al. Early autologous stem cell transplantation (SCT) in genetically poor-risk chronic lymphocytic leukemia is feasible and effective: results from a prospective multicenter study (GCLLSG CLL3 Protocol). Blood. 2004;104:45a.

43. Gribben JG, Zahrieh D, Stephans K, et al. Autologous and allogeneic stem cell transplantations for poor-risk chronic lymphocytic leukemia. Blood. 2005;106(13):4389-96.

44. Gribben JG. Stem cell transplantation in chronic lymphocytic lekemia.. Biol Blood Marrow Transplant 2008;15(suppl 1):53-58.

45. Milligan DW, Fernandes S, Dasgupta R, et al. Results of the MRC pilot study show autografting for younger patients with chronic lymphocytic leukemia is safe and achieves a high percentage of molecular responses. Blood. 2005;105(1):397-404.

46. Darrington DL, Vose JM, Anderson JR, et al. Incidence and characterization of secondary myelodysplastic syndrome and acute myelogenous leukemia following high-dose chemoradiotherapy and autologous stem-cell transplantation for lymphoid malignancies. J Clin Oncol. 1994;12(12):2527-34.

47. Kharfan-Dabaja MA, Anasetti C, Santos ES. Hematopoietic cell transplantation for chronic lymphocytic leukemia: an evolving concept. Biol Blood Marrow Transplant. 2007;13(4):373-85.

48. Michallet M, Archimbaud E, Bandini G, et al. HLA-identical sibling bone marrow transplantation in younger patients with chronic lymphocytic leukemia. European Group for Blood and Marrow Transplantation and the International Bone Marrow Transplant Registry. Ann Internal Med. 1996;124(3):311-5.
49. Doney KC, Chauncey T, Appelbaum FR. Allogeneic related donor hematopoietic stem cell transplantation for treatment of chronic lymphocytic leukemia. Bone Marrow Transplant. 2002;29(10): 817-23.

50. Khouri IF, Przepiorka D, van Besien K, et al. Allogeneic blood or marrow transplantation for chronic lymphocytic leukaemia: timing of transplantation and potential effect of fludarabine on acute graft-versus-host disease. Br J Haematol. 1997;97(2):466-73.

51. Sorror ML, Storer BE, Sandmaier BM, et al. Five-year followup of patients with advanced chronic lymphocytic leukemia treated with allogeneic hematopoietic cell transplantation after nonmyeloablative conditioning. J Clin Oncol. 2008;26(30): 4912-20.

52. Dreger P, Brand R, Hansz J, et al. Treatment-related mortality and graft-versus-leukemia activity after allogeneic stem cell transplantation for chronic lymphocytic leukemia using intensityreduced conditioning. Leukemia. 2003;17(5):841-8.

53. Khouri IF. Reduced-intensity regimens in allogeneic stem-cell transplantation for non-hodgkin lymphoma and chronic lymphocytic leukemia. Hematology. Am Soc Hematol Educ Program. 2006;390-7.

54. Schetelig J, Thiede C, Bornhauser M, et al. Evidence of a graftversus-leukemia effect in chronic lymphocytic leukemia after reduced-intensity conditioning and allogeneic stem-cell transplantation: the Cooperative German Transplant Study Group. J Clin Oncol. 2003;21(14):2747-53.

55. Delgado J, Thomson K, Russell N, et al. Results of alemtuzumabbased reduced-intensity allogeneic transplantation for chronic lymphocytic leukemia: a British Society of Blood and Marrow Transplantation Study. Blood. 2006;107(4):1724-30.

56. Brown JR, Kim HT, Li S, et al. Predictors of improved progressionfree survival after nonmyeloablative allogeneic stem cell transplantation for advanced chronic lymphocytic leukemia. Biol Blood Marrow Transplant. 2006;12(10):1056-64.

57. Moreno C, Villamor N, Colomer D, et al. Clinical significance of minimal residual disease, as assessed by different techniques, after stem cell transplantation for chronic lymphocytic leukemia. Blood. 2006;107(11):4563-9.

58. Dreger P, Brand R, Milligan D, et al. Reduced-intensity conditioning lowers treatment-related mortality of allogeneic stem cell transplantation for chronic lymphocytic leukemia: a populationmatched analysis. Leukemia. 2005;19(6):1029-33.

59. Schetelig J, van Biezen A, Brand R, et al. Allogeneic hematopoietic stem-cell transplantation for chronic lymphocytic leukemia with 17p deletion: a retrospective European Group for Blood and Marrow Transplantation analysis. J Clin Oncol. 2008;26(31): 5094-100.

Este artigo foi avaliado pelos coordenadores das Diretrizes do Transplante de Medula Óssea da Sociedade Brasileira de Transplante de Medula Óssea, Luis Fernando Bouzas, Prof. Julio Cesar Voltarelli e Nelson Hamerschlak, e publicado após avaliação e revisão do editor, Milton Artur Ruiz.

Recebido: 09/10/2009

Aceito: $23 / 11 / 2009$ 